\title{
ERIC WINSBERG Y LA EPISTEMOLOGÍA DE LAS SIMULACIONES COMPUTACIONALES
}

\author{
ERIC WINSBERG AND THE EPISTEMOLOGY OF COMPUTER SIMULATIONS
}

\author{
JUAN M. DURÁN \\ Universidad Nacional de Córdoba - CONICET (Argentina) \\ juanduran@gmail.com
}

RECIBIDO: $24 / 01 / 2017$

ACEPTADO: $27 / 02 / 2017$

Resumen: En este trabajo presento un estudio sobre el estado del arte de la llamada 'epistemología de las simulaciones computacionales'. En particular, me centro en los varios trabajos de Eric Winsberg quién es uno de los filósofos más fructíferos y sistemáticos en este tema. Además de analizar la obra de Winsberg, y basándome en sus trabajos y en el de otros filósofos, mostraré que hay buenas razones para pensar que la epistemología tradicional de la ciencia no es suficiente para el análisis de las simulaciones computacionales.

Palabras claves: simulaciones computacionales, novedad epistemológica, modelos científicos.

\begin{abstract}
In this article, I present a study on the state of the art 'epistemology of computer simulations'. Of particular interest is the work of Eric Winsberg, arguably one of the most fruitful and systematic philosophers on the subject. Apart from the analysis of Winsberg's work, I also submit that there are good reasons for believing that standard philosophy of science is not enough to account for computer simulations.
\end{abstract}

Keywords: computer simulations, epistemological novelty, scientific models.

Eric Winsberg se ha destacado como un autor muy fructífero en materia de producción de trabajos sobre simulaciones computacionales. A diferencia de muchos otros autores, Winsberg sostiene que las simulaciones computacionales no pueden adecuarse a una epistemología tradicional, sino que, más bien, deben considerarse dentro del marco de una epistemología propia. A pesar de que las simulaciones computacionales son fundamentalmente un intercambio de soluciones analíticas por cálculo y que por ello, a primera vista, aparenten ser simplemente una transformación matemática, la pregunta acerca de los grados de confianza que se tiene en el resultado de la simulación va más allá de los grados de confianza en el cálculo y se instala en la totalidad del proceso de construcción, diseño y ejecución de la simulación. Estas son algunas de las razones por las cuales Winsberg cree que un marco epistemológico tradicional no se ajusta a las necesidades de las simulaciones computacionales. 
Por un lado, el cambio de marco epistemológico propuesto es novedoso respecto de la posición de otros autores. Por otro lado, Winsberg comparte con sus contemporáneos la necesidad de aplicar restricciones a los fenómenos simulados reconociendo que aún persiste un espacio de acción científica que no logra ser contenido dentro de los conceptos usuales de la filosofía de la ciencia: "mi discusión", dice Winsberg, "no necesariamente será relevante para simulaciones que no recurren a una base en una teoría aceptada, tales como la simulación de patrones de tráfico o la simulación del vuelo de una bandada de pájaros" (Winsberg 1999: 5).

\section{Pasos previos a una Epistemología de las Simulaciones Computacionales}

Antes de entrar en la explicación de lo que sería propiamente una epistemología de las simulaciones computacionales, será preciso prestar atención a algunos términos técnicos.

Una simulación computacional, en términos generales, comprende un rico proceso inferencial que atraviesa una estructura jerárquica de cinco tipos de modelos diferentes. Nombrados desde la base de la estructura jerárquica -donde se encontrarían los modelos más abstractos-, se dividen en: modelos mecánicos, modelos dinámicos, modelos ad hoc, el modelo computacional y finalmente, en la cima de la jerarquía, el modelo del fenómeno (Winsberg 1999: 3). Cada paso en el proceso de modelar una simulación es una inferencia que va de un modelo a otro en la jerarquía de modelos. A través de estos pasos se espera inferir un nuevo conocimiento acerca del sistema simulado a partir del conocimiento teórico existente.

Cada modelo en la jerarquía de modelos tiene su propio contenido sustantivo y cumple una función determinada. El primer modelo con el cual "comienza" el diseño de una simulación computacional, el modelo mecánico, cumple la función de interactuar entre la teoría y el mundo real. Un modelo mecánico es una suerte de caracterización 'desnuda' de un sistema físico mediante el uso de estructuras teóricas que sirven para asignar una familia de ecuaciones a un sistema. Sin embargo el modelo mecánico es, por sí mismo, una entidad muy general. De aquí que usualmente sea necesario especificar una serie de parámetros, valores y condiciones iniciales, cotas y todo lo necesario para restringir el modelo mecánico a una clase específica de fenómeno: "esta conjunción de teoría con parámetros, cota y condiciones iniciales hace un modelo dinámico concreto (más específicamente, una familia de modelos dinámicos) para una clase de fenómeno muy específica" (Winsberg 1999: 8). Las restricciones, que se exigen en este 
nivel de la jerarquización, por lo general no son logradas directamente y en el primer intento, sino que, más bien, consisten en un delicado balance entre precisión, tratabilidad y experiencia con el fenómeno a modelar.

El siguiente modelo es el denominado modelo computacional. Algunos modelos dinámicos ${ }^{1}$ son analíticamente tratables, ya que las ecuaciones diferenciales que contienen pueden ser resueltas. Este caso cubre, si recordamos bien, la segunda crítica de Hartmann a Humphreys (Hartmann 1996): las simulaciones pueden llegar a ser interesantes mecanismos de aproximación a fenómenos, aun cuando están disponibles soluciones analíticas. Sin embargo debemos reconocer que el caso paradigmático y que más interesa al científico es aquel donde las soluciones analíticas no se encuentran disponibles. Allí es donde entran en escena los modelos computacionales: los modelos mecánicos necesitan ser trasformados en un modelo computacional a fin de que se puedan implementar en una computadora. Diferentes técnicas computacionales, tanto teóricas como creativas, intervienen en la configuración, construcción y traspaso a este modelo.

El paso de los modelos dinámicos a los modelos computacionales debe ser realizado en dos etapas. La primera consiste en convertir las ecuaciones diferenciales del modelo dinámico en ecuaciones discretas, para las cuales la computadora debe encontrar distintas soluciones. A pesar de que esto resuelve el problema de la intratabilidad analítica, puede darse el caso de que el nuevo modelo sea computacionalmente intratable. Como se sabe, la capacidad de cálculo de una computadora digital se ve seriamente afectada a medida que mejoramos la precisión de cálculo, todo lo cual indica que la precisión en los resultados será un contexto a tener presente por los diseñadores, ya que no querrán intercambiar el problema de la intratabilidad analítica por el de la intratabilidad computacional. El paso de los modelos dinámicos a modelos computacionales es, como puede apreciarse, sensible a las posibilidades tecnológicas vigentes.

La segunda etapa consiste en un tratamiento de superación de la primera etapa. Para ello Winsberg presenta los modelos ad hoc. Estos modelos son el resultado de la implementación de diversas técnicas de diseño sobre los otros niveles de la estructura. Incluyen simplificaciones, eliminación de grados de libertad, sustitución de relaciones teóricas simples por complejas -y viceversa-, etc. Para organizar estas diversas técnicas, Winsberg traza una pequeña

\footnotetext{
1 Es oportuno mencionar aquí que Winsberg no presta excesiva atención a la distinción, que tanto le preocupa a Simpson (2006) y Hartmann (1996), por ejemplo, entre 'modelos' y 'modelos dinámicos'. Se sugiere, en todo caso, que los modelos que constituyen el corazón de la simulación computacional deben ser, por definición, modelos dinámicos.
} 
categorización: modelos ad hoc eliminativos y modelos ad hoc creativos. El primer tipo de modelo ad hoc sirve para determinar si los factores que han sido descartados en el diseño de la simulación pueden ser ignorados; el segundo tipo consiste en hacer uso de algún "factor de improvisación" [fudge factor] para compensar aquellos factores negados que, usualmente por su complejidad, no han podido ser modelados. La idea de introducir modelos ad hoc es crucial: consiste en enfatizar el hecho de que la construcción de la simulación computacional descansa en un entendimiento logrado desde fuera del contexto de nuestro mejor entendimiento teórico del fenómeno, esto es, desde elementos creativo-inferenciales y no deductivo-teóricos.

Un ejemplo que utiliza Winsberg para ilustrar estas ideas es el de la simulación de una estrella gigante roja (Winsberg 2001). Este caso resulta interesante puesto que la estrella es particularmente compleja e inestable. Modelar una estrella gigante roja requiere de algunas consideraciones por parte de los científicos: pequeños cambios en temperatura, presión y densidad en una parte del sistema podría desembocar en turbulentos vórtices en otros puntos del sistema; y pequeños remolinos en la superficie pueden llevar a grandes flujos muy conflictivos (Winsberg 2001: 445). Todo esto da la idea de que, si el modelo es pensado para capturar los efectos a gran escala, también debe tener presente los efectos a pequeña escala sin que esto signifique el desborde de las capacidades de cálculo en los cómputos. Por el otro lado, las ecuaciones básicas que gobiernan este modelo son las ecuaciones de Euler para fluidos dinámicos. Estos son formas de ecuaciones relativamente simples y se basan en leyes de conservación de la masa, momento y energía. En este sentido, el uso de la ecuación de Euler de fluidos "no-viscosos" es un ejemplo de modelado ad hoc eliminativo. A pesar de que la viscosidad se encuentra presente en el flujo del gas de la estrella y contribuye a la dinámica del sistema de modo crucial, esta presencia se da en escalas muy pequeñas al punto de que no suelen ser capturadas, inclusive por programas computacionales muy razonables y eficientes. En este caso el investigador debe hacer uso del modelado ad hoc creativo compensando con algún factor que reemplace el que ha sido eliminado, v. gr. la viscosidad en el flujo del gas de la estrella. Se asume, desde luego, que el investigador realiza gran cantidad de suposiciones que contribuyen a mejorar el modelo del fenómeno.

Por último, el fin de esta estructura jerárquica es lograr un modelo del fenómeno. Una simulación, una vez realizada y finalizada, produce como resultado una gran cantidad de datos que pueden ser representados de distintos modos: estructuras de datos como vectores, arreglos [arrays] o tal vez algo más comprensible a simple vista como puede ser un gráfico - estático o dinámico-: 
Un modelo de un fenómeno es una representación múltiple que expresa el conocimiento relevante, reunido a partir de todas las fuentes relevantes, acerca del fenómeno. Puede consistir en relaciones matemáticas y leyes, imágenes -estáticas o dinámicas-, y descripciones textuales (Winsberg 1999: 11).

La intervención de un observador competente es crucial en tanto es preciso interpretar y calibrar la información resultante. Los aspectos interpretativos no sugieren carga teórica puesto que, según el autor, no hay que tomarlos en un sentido filosóficamente cargado. El caso de las calibraciones es un poco más delicado. Winsberg señala que se pueden realizar de tres modos diferentes: comparando los resultados de una simulación con un experimento real; mediante análisis de los resultados; o mediante la comparación con otras simulaciones. Cada uno de estos modos de calibrar los resultados de una simulación parecería sugerir que el modelo del fenómeno es, en cierto sentido, un producto virgen del que no tenemos demasiado conocimiento. Esta sugerencia se encuentra presente a lo largo de los trabajos del autor, quien sugiere la existencia de una constante retroalimentación entre la simulación -o mejor, entre cada uno de los niveles de la jerarquía-, el experimentador, los aspectos más conocidos de las teorías y, si tenemos acceso, los valores esperados. Todo ello, girando en derredor de un observador competente.

De nuevo, son estas las ideas que llevan a Winsberg a considerar que el marco teórico que podría proporcionar una filosofía de la ciencia sería insuficiente. Pero ¿en qué consiste una 'epistemología de la simulación’? ¿Es, acaso, un nuevo nombre para viejos modos de pensar?

\section{Una epistemología de las simulaciones computacionales}

Hemos mencionado que el diseño y construcción de una jerarquía de modelos sobre la que se basa una simulación computacional consiste en un proceso inferencial no deductivo. Hemos mencionado también que gran cantidad de elementos que contribuyen a esta construcción provienen de fuentes externas a las teorías que dan forma a los primeros modelos. Por último, se ha sugerido que el modelo del fenómeno debe ser sometido a calibraciones que también utilizan técnicas poco ortodoxas. Estas tres características, hay que confesarlo, dan cierta sensación de fragilidad a todo el asunto de las simulaciones. No hay pasos en ninguna etapa de la vida de la simulación que no estén anclados, de un modo u otro, en vicisitudes que obligan a estimular la creatividad de los investigadores. 
Y no es que la creatividad sea una muestra de fragilidad, pero hay que recordar que hasta aquí todos los autores mencionados han esperado dar con una caracterización de las simulaciones computacionales sin tener la necesidad de prestar atención a una metodología de diseño y construcción. El extraño caso de Winsberg tiene que ver con el hecho de que no existen pasos obligatorios ni seguros. Y es allí donde encontraremos una caracterización apropiada.

Pues bien, aceptar la complejidad metodológica en la estructura jerárquica de una simulación computacional significa también preocuparse por la justificación de las conclusiones alcanzadas en una simulación. La influencia de cada modelo, así como sus fallas, deben ser comprendidas y manejadas correctamente, caso contrario representan una amenaza potencial a la credibilidad en los resultados. La comprensión y manejo de los diversos factores involucrados en todo el proceso requiere, antes que nada, haber logrado ciertos grados de confianza aceptables en las fuentes a las que se echa mano. Pero también se requiere de confianza en las aptitudes de los usuarios y diseñadores, en especial cuando gran parte de este conocimiento no se encuentra contenido en las estructuras formales que establecen las bases originales de la simulación. En definitiva, a pesar de que las simulaciones conciernen a la sustitución de soluciones analíticas por cálculo, la pregunta acerca de la confianza en los resultados de la simulación va más allá de las preocupaciones por el crédito otorgado al cálculo, remontándose tanto al nivel del proceso de simulación en su totalidad como a las conclusiones alcanzadas.

Para Winsberg, el modo en el que hemos visto cómo se constituyen las simulaciones computacionales exigen de una epistemología propia. Persiguiendo estos fines, el autor se centra en tres características de esta nueva epistemología de las simulaciones computacionales, a saber: descendentes [downward], autónomas y variadas [motley].

La primera de estas características se asocia al método con el que son diseñadas las simulaciones computacionales. Por regla general, una simulación parte de una teoría y, a medida que se avanza de un modelo a otro, se van "quitando" grados de abstracción y generalidad. Es en este sentido que una epistemología de las simulaciones debe tener en cuenta una metodología descendente $^{2}$. Esta epistemología consiste en tener que justificar inferencias de la teoría a la aplicación -inferencias que los filósofos de la ciencia asumen como deductivas y que acarrean la justificación de los niveles superiores a los inferiores. En efecto, la construcción de los modelos son guiados, pero no

\footnotetext{
2 Descendente en el sentido de que parte de los modelos mecánicos a aproximaciones razonables de fenómenos concretos (modelos computacionales más modelos ad hoc).
} 
determinados, por la teoría. La credibilidad de una simulación proviene no solo de las credenciales suplidas por la teoría que gobierna la simulación, sino también de las credenciales epistémicas suplidas por cada uno de los modelos que conforman una simulación. En otras palabras, a medida que los modelos van especificándose, ciertas credenciales epistémicas son heredadas por los modelos más específicos.

La segunda de las características es más sutil. Winsberg considera que usualmente, cuando queremos conocer si una estructura representacional es precisa o confiable, se la compara con aquello que representa. Este es un método usual en los filósofos de la ciencia. Así, si queremos convencernos de que la estructura de una teoría es una buena interpretación del fenómeno que representa, simplemente debería 'comparársela' con la estructura en la que se articula el fenómeno. El caso es que las técnicas de supervisado de las simulaciones computacionales (cuando están disponibles) son del todo diferentes a las que encontramos, por ejemplo, en la experimentación de laboratorio. En particular, es usual que las simulaciones sean requeridas justamente cuando la información de un fenómeno se encuentra dispersa -o directamente carecemos de ella- y por lo tanto no contamos con el factor 'comparación directa con el fenómeno'. Las simulaciones que son utilizadas para estudiar la estructura interna de una estrella, por ejemplo, o para determinar la distribución de la presión del viento dentro de una tormenta, no cuentan, ab initio, con toda la información necesaria.

Si fuera posible confirmar una simulación fuera de ella misma, esto es, confrontarla directamente con el fenómeno que simula o con el puro análisis, podríamos decir que ha sido justificada de forma dependiente - pues depende de un correlato para ser justificada. Pero este usualmente no es el caso. El proceso de transformar un modelo teórico en un modelo computacional tratable debe ser justificado de modo independiente, esto es, atendiendo a su forma interna y no solo sobre la base de una comparación del resultado de la simulación y el fenómeno. Las simulaciones requieren, así, una epistemología que permita la evaluación de la confiabilidad en los resultados y del modelo. En términos más generales, las transformaciones internas de una simulación deben ser valoradas en el contexto de toda la simulación y no solo en sus resultados; los pasos inferenciales deben ser evaluados en diferentes frentes, atendiendo a consideraciones provenientes de la teoría, de generalizaciones empíricas, de datos o por experiencia previa en modelar fenómenos similares en otros contextos. Esta es precisamente la segunda caracterización de la epistemología de las simulaciones, a saber, su autonomía.

El último aspecto de los rasgos de esta epistemología es que las simulaciones computacionales son consideradas variadas [motley]. A pesar de que usualmente 
se toman las simulaciones como mecanismos de resolución de ecuaciones matemáticas y que para estas ecuaciones contamos con suficiente conocimiento, el aporte de conocimiento teórico neto que realiza el científico corresponde solo a una porción de los ingredientes que intervienen en una simulación. Existe, empero, gran cantidad de elementos que deben ser considerados como fuente de constitución y de sopesamiento de los resultados de una simulación. La credibilidad de la simulación se sustentará en diversas fuentes tales como habilidades adquiridas con experiencias pasadas, reutilización de mecanismos aplicados en otras simulaciones exitosas, sugerencias de colegas, etc. En definitiva, no todas las fuentes que componen una simulación están teóricamente articuladas. Un ejemplo que ayudará a comprender este alejamiento de un esquema estrictamente fundamentado es el de una simulación que emplea celdas discretas, tal y como se da en modelos de comunicación móvil, donde es muy importante tener presente que es preciso solucionar problemas 'locales', v. gr. en cada celda, pero que el producto final consiste en la articulación de toda esta información local recolectada. Sin embargo muchas veces no se cuenta con elementos teóricos que dictaminen qué función matemática debe ser aplicada para "recolectar" la información local de las celdas o cómo articularlas con el resto del sistema. En estos casos el científico que esté llevando a cabo la simulación deberá tomar una decisión acerca de cómo calcular, interpretar y articular esta información. Casos como estos son los que inclinan a Winsberg a ver con cierta preocupación que los filósofos estén más focalizados en pensar cómo caracterizar una simulación computacional en términos estrictamente teóricos antes que intentar un acercamiento más práctico.

Estas tres características, como dijimos, son constitutivas de una epistemología de las simulaciones computacionales según Winsberg. Como puede apreciarse, además intentan presentar, en términos sucintos y generales, lo que se debería tener presente, si se quisiese practicar una justificación de las simulaciones computacionales. Desde luego esta es una empresa bastante más compleja de lo que Winsberg está dispuesto a reconocer en principio, por lo cual demandar una nueva epistemología parecería, al menos, prematuro. De hecho, aceptarle a Winsberg que las simulaciones tienen su propia epistemología y que esta es descendente, autónoma y variada nos pone, automáticamente, en un compromiso muy grande con la tradición filosófica. Sobre estas objeciones a una nueva epistemología liderada por las simulaciones computacionales volveré en la siguiente sección. Permítaseme cerrar ahora el análisis de las tesis centrales del autor.

De nuevo, las ecuaciones que forman las bases teóricas para algunos sistemas de interés pueden resultar analíticamente irresolubles. En otras palabras, no 
siempre se cuenta con una expresión matemática que dé la solución de esas ecuaciones. De aquí que, para Winsberg, ninguna representación formal de estos sistemas puede darse en forma de entidades lingüísticas derivables de estructuras teóricas. Así pues, parecería que Winsberg maneja una versión moderna de modelo, el cual es autónomo tanto de la teoría como del mundo (Morgan y Morrison 1999). Sin embargo, los procedimientos deductivos que esta concepción acepta y que, por definición, confieren certeza a las conclusiones obtenidas acerca de los modelos parecen estar ausentes en la caracterización que Winsberg ha hecho sobre simulaciones computacionales. Recordemos que dentro del marco de la epistemología de la simulación, el proceso inferencial no es deductivo sino que, en el mejor de los casos, confiere alguna garantía débil pero razonable para creer las conclusiones que se alcanzan, y esto solo si se ha sido cuidadoso en los pasos realizados. Se podría argüir, empero, que mientras los resultados de una simulación no son deducibles de la teoría, podrían sin embargo mantener una relación lógica con ella. Nuevamente Winsberg recuerda que el hecho de que no exista una certeza fuerte en el proceso inferencial es un síntoma de que hay posibilidades de que algo salga mal y de que nuestros resultados no mantengan ninguna relación lógica con la teoría. Creo, empero, que Winsberg podría radicalizar sus ideas aquí y no circunscribirlas a la posibilidad de que algo salga mal. Mientras pensemos las simulaciones como "productoras" de nuevo conocimiento, es posible que, surgiendo de una teoría - de un conjunto de teorías-, lleguemos a resultados que habrían sido impredecibles por estas teorías sin la contribución de todos los elementos intermedios que participan en el proceso de diseño y construcción de la simulación. En otras palabras, los vínculos que el resultado de una simulación puede mantener con cualquier modelo anterior no parecería estricto ni determinante como lo pide, en general, la concepción de modelos como mediadores.

A pesar de que esta forma de caracterizar la situación puede entenderse como excesivamente vaga y con poco detalle, aun así permite realizar una pregunta central: ¿tenemos bases para creer que las simulaciones computacionales son buenas aproximaciones a estructuras del sistema objeto (sea empírico o de otro tipo) que se pretenden simular? Dada la rica complejidad de los procesos que intervienen en las simulaciones computacionales y la focalización de estos en elementos externos a cualquier estructura teórica o modelística, sería poco acertado interpretar que estos procesos implementan una representación estricta del sistema objeto. Si escudriñamos con cuidado los pasos epistemológicos que se necesitan para dar alguna garantía a todo el proceso de la simulación, en especial a su resultado, se verá que tienen que ver más con cuidar que los resultados se asemejen lo mejor posible al sistema objeto, que con asegurarse de 
que estos resultados se parezcan a una estructura. En otras palabras, la confianza que se deposita en los resultados de las simulaciones depende en gran medida de los factores que han sido puestos en juego, ninguno de ellos garantizado por conocimiento teórico o modelístico. Depende, entonces, de hechos que conciernen a nuestras computadoras y a nuestras técnicas gráficas. Depende de los lenguajes de programación y de conocimiento experto. Depende de la confianza que tenemos en varios de los modelos ad hoc que utilizamos confianza que suele depender, a su vez, de la experiencia obtenida en el laboratorio y en la observación. Depende también de nuestra habilidad para calibrar los modelos con resultados empíricos, cuando esto es posible. Depende también del hecho de contar con una historia de éxitos en el uso y práctica con una simulación computacional. Y finalmente depende de la confianza tácita que tenemos en nuestras habilidades como observadores y como intérpretes de la información resultante de la simulación. La epistemología de la simulación, como hemos podido ver, se parece mucho a lo que podríamos llamar una epistemología empírica -o práctica-, una forma de hacer epistemología que está en gran parte ausente en el trabajo de Winsberg.

Esta epistemología de la simulación traza el marco teórico de lo que es una simulación computacional: todo lo que entre en este marco será una simulación, aunque no podamos dar con una definición teórica que lo clausure. Apelando al espíritu de esta epistemología, una simulación computacional será algo semejante a lo que la práctica científica dice que es, y esto no es otra cosa que dar una definición ostensiva: "esto es una simulación" quiere decir que se emplean determinados pasos inferenciales, que se hace uso de distintos tipos de modelos, de criterios de diseño y construcción, y que se esperan ciertos resultados que podrían representar un fenómeno. Y eso es todo. El resto quedará en manos de un individuo competente que pueda diferenciar una simulación computacional de una hoja de cálculo, de un ábaco, o de un cálculo hecho con papel y lápiz.

\section{¿Nuevos nombres para viejos modos de pensar?}

En un trabajo del 2008, Roman Frigg y Julian Reiss escribieron un artículo en el cual analizaban la relevancia de las simulaciones computacionales en la práctica científica y cuestionaban fuertemente dichos como los de Winsberg con respect a la necesidad de una nueva epistemología. Para estos dos autores hay un interés exagerado $\mathrm{y}$, en principio, infundado sobre el uso de simulaciones computacionales en la práctica científica contemporánea. A esto le sigue una 
fingida demanda por una nueva epistemología de la ciencia. Frigg y Reiss indican que estas dos aserciones se encuentran en Galison (1996), Winsberg (1999, 2001), Humphreys (2004), Rohrlich (1991) y varios otros autores. En este trabajo he mostrado que Winsberg sugiere una nueva epistemología para las simulaciones computacionales, pero dudo que el autor extrapole esto a toda la filosofía de la ciencia. Lo mismo ocurre con los otros autores mencionados por Frigg y Reiss, quienes parecerían no estar diciendo lo que se les adscribe. En cambio, me parece que lo que se está indicando es que el análisis filosófico de las simulaciones computacionales requiere de una epistemología distinta de aquella con la que ya se cuenta, que evite así subsumir este análisis filosófico a un subcapítulo de una filosofía más familiar, como lo sería una filosofía de los modelos o una filosofía de la experimentación. En pocas palabras, las epistemologías más conocidas, ya sea provenientes de los modelos científicos o de la experimentación de laboratorio, no darían cuenta de las simulaciones computacionales. En particular, Galison sugiere que el interés filosófico en simulaciones computacionales descansa en los cambios ontológicos que estas imponen con respecto a la práctica científica más tradicional. Otro buen ejemplo de esto es el mismo Winsberg quien, como ya he mencionado, sostiene que la compleja cadena de inferencias que transforman estructuras teóricas en conocimiento concreto de sistemas físicos es central en la epistemología de las simulaciones computacionales. Nada de esto, en principio, conlleva tesis fuertes como que la epistemología de la ciencia o la filosofía de la ciencia deben ser reescritas a la luz de las simulaciones computacionales.

Sin embargo, es siempre un ejercicio intelectual saludable el exteriorizar dudas sobre la importancia de estudiar filosóficamente nuevos métodos de obtención de conocimiento científico. Para esto, Frigg y Reiss basan su caso en las cuatro tesis siguientes:

Metafísica: Las simulaciones crean algún tipo de mundo paralelo en el cual los experimentos pueden ser controlados en condiciones más favorables que en el 'mundo real'.

Epistémico: Las simulaciones demandan una nueva epistemología.

Semántico: Las simulaciones demandan un nuevo análisis de cómo los modelos/teorías se relacionan con fenómenos concretos.

Metodológico: Simular es una actividad sui generis que está 'entre' teorizar y experimentar (Frigg y Reiss 2009: 595)

De acuerdo con los autores, entonces, los filósofos, al trabajar en simulaciones computacionales, han construido y basado sus argumentos en una u otra de estas cuatro tesis, ninguna de las cuales aboga por la idea de una "nueva 
epistemología" en el sentido que le quieren dar los autores. De hecho, Frigg y Reiss afirman que las simulaciones computacionales "sugieren pocos problemas filosóficos" (Frigg y Reiss 2009: 594) y que por lo tanto cualquier asunto relacionado con ellas es de otro orden, ya sea matemático, físico, computacional, o incluso psicológico, pero definitivamente no filosófico.

En este punto es donde las opiniones comienzan a divergir. Humphreys, por ejemplo, responde al artículo de Frigg y Reiss defendiendo la idea de que las simulaciones computacionales son, de hecho, algo novedoso en la práctica científica que introduce cuestiones de genuino interés filosófico. En particular presenta lo que él llama el predicamento antropocéntrico: “¿cómo podemos nosotros, como humanos, entender y evaluar métodos científicos basados en computación y que trascienden nuestras habilidades?" (Humphreys 2009: 616). El predicamento antropocéntrico ha sido diseñado para cuestionar una filosofía de la ciencia marcadamente empirista, en cuyo centro sigue estando el hombre y su capacidad cognitiva de observar y experimentar ${ }^{3}$. En cambio, las simulaciones computacionales son utilizadas como "métodos que empujan a los seres humanos del centro de la empresa epistemológica" (Humphreys 2009: 616), convirtiendo de este modo el estudio de las simulaciones computacionales en una empresa de interés filosófico en sí mismo.

El trabajo de Humphreys no fue solo una respuesta a Frigg y Reiss, sino que además contiene el argumento de Winsberg discutido en la sección anterior. En efecto, Humphreys muestra que sí hay elementos para pensar en la necesidad de una nueva epistemología exclusiva de las simulaciones computacionales. El predicamento antropocéntrico es, sin lugar a duda, privativo de las simulaciones computacionales como método de producción de conocimiento. En este sentido, las simulaciones computacionales abren la puerta para repensar (aunque no necesariamente reemplazar) varias tesis más o menos bien acentuadas en la tradición filosófica. Como ejemplo de esto último, uno puede pensar la noción de 'dato'. Barberousse, Franceschelli e Imbert (2009), por ejemplo, han trabajado esta línea de argumentación. Para estos autores, las nociones tradicionales de dato, fuertemente ancladas en la experimentación científica como recolección de información mediante medición y observación, no permitirían la correcta conceptualización del dato computacional.

Simplificando su posición, Barberousse et. al, dividen la noción de dato en dos tipos: datos $\mathrm{E}_{\mathrm{E}}$ (datos empíricos) y datos $\mathrm{A}_{\mathrm{A}}$ (datos sobre un sistema). Los primeros son datos producidos por la interacción física mediante dispositivos de

\footnotetext{
3 Humphreys insiste que, aun en pleno auge de una filosofía Lógico-Positivista donde la psicología fue desplazada, el hombre continuó siendo el centro de producción de conocimiento (Humphreys 2009: 616).
} 
detección, como puede ser la medición del péndulo que mide la fuerza gravitacional $g$. Los datos ${ }_{\mathrm{A}}$, por el otro lado, son entendidos como datos sobre un sistema físico, como $9.8 \mathrm{~m} / \mathrm{s}^{2}$ es un dato sobre la fuerza $g$. Como podría esperarse, estos dos tipos de datos no son necesariamente disjuntos, ya que los datos sobre un sistema $\left(\right.$ datos $\left._{\mathrm{A}}\right)$ pueden tener un origen empírico (datos $\mathrm{E}_{\mathrm{E}}$ ). El ejemplo de medir la fuerza gravitacional $g$ es precisamente un caso de esto último. Sin embargo, datos ${ }_{\mathrm{A}}$ tienen la característica de que pueden ser obtenidos por medios de puro cálculo, ya sea con papel y lápiz o con computadoras (Barberousse et al. 2009: 560). Hasta ahí, podría argumentarse, no hay nada de novedoso en la noción de dato. Sin embargo el giro está en el hecho de que, después de analizar los datos obtenidos de simulaciones computacionales, los autores concluyen en que la línea que divide datos de simulaciones computacionales de datos empíricos puede ser dibujada precisamente con una base semántica. El análisis semántico, pues, se focaliza primariamente en dos elementos, a saber, en el origen de producción de datos y en la capacidad representacional sobre las propiedades del sistema objeto. De acuerdo con los autores, estos dos elementos forman la novedad que subyace en la noción de dato, donde el origen es computacional y está fuera de todo alcance cognitivo, y la capacidad representacional juega un papel central en la validación de dichos datos.

Estas ideas, correctas o no, son las que dan forma a la idea de una nueva epistemología para las simulaciones computacionales defendida, entre otros, por Winsberg, Humphreys, Barberousse et. al., y desdeñada por Frigg y Reiss.

A pesar de que el tipo de simulaciones escogidas por Winsberg comienzan, fundamentalmente, con un modelo teórico, y a pesar de que se tome las simulaciones como un intento de resolver ecuaciones matemáticas, el conocimiento teórico que tenemos será considerado solo uno de varios ingredientes que se usan para producir resultados de las simulaciones computacionales. Así pues, para Winsberg la relación que la teoría mantiene con los modelos es que "nuestra confianza en nuestras teorías físicas es una de las cosas que, en combinación con otros elementos, garantiza nuestra creencia racional de que los modelos que construimos son fiables y cognitivamente útiles" (Winsberg 1999: 25). Solo si vemos las simulaciones como un intento de proveer representaciones de sistemas reales, y no simples modelos abstractos, la epistemología de las simulaciones computacionales cobra sentido.

La insatisfacción de estas ideas puede estar anclada en el hecho de que se esperaba que, tal vez, existiera una relación más directa y dependiente entre el resultado de las simulaciones computacionales y la teoría. Los modelos que se construyen necesitan ser cuidadosa y delicadamente confeccionados y para ello 
existen muchos mecanismos, formas y herramientas disponibles: “consecuentemente, esos modelos no son meras 'soluciones' a nuestras estructuras teóricas. A pesar de que son el resultado de una forma de cálculo, son ricos constructores que median entre nuestras teorías y el mundo" (Winsberg 1999: 26).

Estas últimas palabras de Winsberg quedan resonando, ya que son lo suficientemente familiares como para ponernos en alerta. Es tal vez el momento de comparar las conclusiones del autor con algunos puntos cruciales de las ideas de Morgan y Morrison. Los modelos como instrumentos de mediación han sido construidos a fin de extender nuestras teorías en nuevos dominios de aplicación. En la práctica, las teorías proveen guías de cómo esos modelos deberían ser construidos pero no determinan su forma final. En este sentido los modelos no son derivados de la teoría, pero tampoco dirigidos por los datos. La concepción semántica puede ser criticada dado que impide ver cómo una teoría puede guiar una aplicación en un área que previamente no estaba en su dominio.

Al ocuparnos de sistemas muy complejos, partimos de conocimiento teórico y nos movemos hacia la obtención de nuevo conocimiento y de nuevas representaciones. De aquí que los modelos que necesitamos construir deben ser confeccionados delicadamente y derivados de diversas fuentes, puesto que no es posible tomar solo la teoría como único aporte a dicha construcción. Consecuentemente, esos modelos no son meras instancias de nuestras estructuras teóricas, sino más bien son el resultado de una pluralidad de contribuciones, cada una de las cuales proporciona diferentes elementos al modelo. La lección que hay que aprender, entonces, es que necesitamos movernos hacia una filosofía de la ciencia que se focalice en modelos concretos antes que en teorías abstractas, como locus de representaciones confiables de sistemas reales. Entender cómo son construidos esos modelos, cómo funcionan en su capacidad representacional y de dónde obtienen su confiabilidad es un importante desafío para la filosofía de la ciencia. A su vez, las prácticas de modelado en la ciencia son diversas, pero además de ser diversas, deben ser realizadas en capas, una sobre la otra. Estas son, a mi modo ver, las lecciones centrales de la lectura de Winsberg sobre simulaciones computacionales. Y es precisamente aquí donde descansa la idea de que las simulaciones requieren de una nueva epistemología.

\section{BIBLIOGRAFÍA}

Barberousse, A., Franceschelli, S. y Imbert, C. (2009). "Computer Simulations as Experimenta" Synthese 169: 557-574 
Frigg, R., Reiss, J. (2009). "The Philosophy of Simulation: Hot New Issues or Same Old Stew?" Synthese 169: 593-613.

Galison, P. (1996) "Computer Simulation and the Trading Zone" en Disunity of Science: Boundaries, Contexts, and Power. Galison, P. y Stump, D. (comp.). Stanford University Press, (1996: 118-157).

Hartmann, S. (1996) "The World as a Process: Simulation in the Natural and Social Sciences" en Simulation and Modelling in the Social Sciences from the Philosophy of Science Point of View. Hegselmann, R. Müller, U., y Troitzsch, K. (comp.) Kluwer, (1996: 77-100).

Humphreys, P. (1990). “Computer Simulations” PSA 2: 497-506.

Humphreys, P. (2004). Extending Ourselves. Computational Science, Empiricism, and Scientific Method (Oxford University Press).

Humphreys, P. (2009). "The Philosophical Novelty of Computer Simulation Methods" Synthese 3: 615-626.

Morgan, M. y Morrison, M. (1999). Models as Mediators. Perspectives on Natural and Social Sciences (Cambridge University Press).

Rohrlich, F. (1990) "Computer Simulation in the Physical Sciences" PSA: 507818.

Simpson, J. (2006) "Simulations are not Models" Proceedings Models and Simulations, London, 2006 1-33.

Winsberg, E. (1999). "Sanctioning Models: The Epistemology of Simulation" Science in Context 12 (2): 275-292.

Winsberg, E. (2001). "Simulations, Models, and Theories: Complex Physical Systems and Their Representations" Philosophy of Science 68: 442-454.

Winsberg, E. (2003). "Simulated Experiments: Methodology for a Virtual World" Philosophy of Science 70: 105-125.

Winsberg, E. (2006). "Models of Success versus The Success of Models: Reliability without truth" Synthese 152: 1-19. 
